Palavras chave: Inventário Florestal Unidade amostral Forma da parcela

Histórico:

Recebido 16/06/201 I

Aceito $31 / 08 / 2014$

Keywords:

Forest inventory

Sample unit

Plot shape

Correspondência: geronimendes@gmail.com
Rodrigo Geroni Mendes Nascimento, Luís César Rodrigues da Silva, Laércio da Silveira Soares Barbeiro, Julio Cesar Wojciechowski, Sylvio Péllico Netto, Sebastião do Amaral Machado

\section{EFEITO DA ÁRVORE MARGINAL NOS ESTIMADORES POPULACIONAIS OBTIDOS POR MÉTODOS DE AMOSTRAGEM DE ÁREA VARIÁVEL}

RESUMO: Nesta pesquisa, objetivou-se avaliar o efeito da árvore marginal nos estimadores populacionais obtidos pelos métodos de amostragem de Bitterlich (1948), Prodan (1968) e Strand (1958). A base de dados foi um censo realizado em fragmento de Floresta Ombrófila Mista Montana situado no Campus III da UFPR, Curitiba - PR. Todas as árvores com DAP $\geq 10 \mathrm{~cm}$ foram medidas, identificadas, georreferenciadas, e consideradas como possíveis centros das unidades amostrais dos métodos. De todas as árvores, 185 foram sorteadas, aleatoriamente, como início dos pontos amostrais para a estimativa de $\mathrm{N} \cdot \mathrm{ha}^{-1}, \mathrm{G} \cdot \mathrm{ha}^{-1}$ e $\mathrm{V} \cdot \mathrm{ha}^{-1}$ para os três tratamentos distintos: sem a influência da árvore marginal, contagem de meia árvore e contagem da árvore marginal corrigida pelo Fator P de Péllico Netto (1994). Independente do método e do tratamento utilizado ocorreu superestimativa do $\mathrm{N} \cdot \mathrm{ha}^{-1}$. Para a estimativa da área basal, bem como do volume por hectare, o método de Bitterlich apresentou os melhores resultados, seguido de Strand e Prodan, respectivamente. $\mathrm{A}$ aplicação do fator $\mathrm{P}$ nas árvores marginais não melhorou expressivamente os estimadores populacionais a ponto de se diferenciar satisfatoriamente da estimativa gerada com as árvores marginais, contadas como meia árvore ou sem a sua influência.

\section{EFFECT OF BORDERLINE TREES IN POPULATION PARAMETERS ESTIMATED BY VARIABLE SAMPLING AREA METHODS}

ABSTRACT: The objective of this research was to evaluate the effect of the borderline tree in the population parameters estimated by Bitterlich (1948), Prodan (1968) and Strand (1958) sampling methods. The database came from a census carried out in a fragment of Mixed Ombrophylous Montana Forest located in the Campus III, of Federal University of Parana, Curitiba-PR, Brazil. All trees with $\mathrm{DBH} \geq 10 \mathrm{~cm}$ were measured, identified, georeferenced, and considered as possible plot center of the sampling units in each method. The sampling simulation was conducted with 185 randomly selected points for the estimation of $\mathrm{N} \cdot \mathrm{ha}^{-1}, \mathrm{G} \cdot \mathrm{ha}^{-1}$ and $\mathrm{V} \cdot \mathrm{ha}^{-1}$ to three different treatments: without the influence of borderline tree, count half borderline tree and count of partial borderline tree corrected by the P factor introduced by Péllico Netto (1994). Regardless of the method and the treatment used there was always an overestimation of $\mathrm{N} \cdot \mathrm{ha}^{-1}$. To estimate the basal area and volume per hectare, the Bitterlich method achieved the best results, followed by Strand and Prodan, respectively. Application of $\mathrm{P}$ factor in borderline trees did not cause a significant improvement in the population estimators compared with the estimates generated by borderline trees counted as half a tree or without its influence. 


\section{INTRODUÇÃO}

Em virtude de restrições de custos, tempo, mão-de-obra, acesso e tamanho, o conhecimento do todo de uma floresta é, em muitas ocasiões, impraticável. Para suprir essa demanda, métodos e processos de amostragem foram desenvolvidos visando à minimização dessas restrições operacionais do manejador florestal.

A amostragem de uma população florestal pode ser realizada segundo diferentes métodos. $O$ mais conhecido é o Método de Área Fixa, chamado assim por possuir limites físicos fixos, em geral com formato circular, quadrado ou retangular. Já, os demais possuem área variável, e foram concebidos por diferentes autores, tais como: Bitterlich (1948), Prodan (1968) e Strand (1958), entre outros.

Esses métodos selecionam as árvores da parcela com probabilidade proporcional a um tamanho, sendo que, na concepção teórica das técnicas de área variável de Bitterlich (1948) e Strand (1958), a contagem ou não de cada indivíduo é feita de forma dicotômica. Os métodos de área variável assumem que não existe "meia árvore" na floresta, com exceção a técnica desenvolvida por Prodan (1968).

Porém, na prática muitos levantamentos feitos por esses métodos, as árvores duvidosas, ou seja, as árvores de bordadura ou do limite do raio variável são contadas como meia árvore, podendo afetar todos os estimadores da população florestal $\mathrm{e}$, consequentemente, $\circ$ resultado das atividades dependentes do inventário florestal.

Objetivou-se, neste trabalho, avaliar os estimadores populacionais obtidos pelos métodos de Bitterlich (1948), Prodan (1968) e Strand (1958), frente aos parâmetros populacionais de um fragmento de Floresta Ombrófila Mista Montana e comparar o desempenho dos métodos, após a correção das árvores marginais, quando contadas parcialmente.

\section{MATERIAL E MÉTODOS}

\section{Área de Estudo}

O presente trabalho foi realizado em um fragmento de floresta conhecido como "Capão da Engenharia Florestal", situado no Campus III Jardim Botânico da Universidade Federal do Paraná. A área do capão é de 15,4 ha, dos quais 13 hectares são ocupados por Floresta Ombrófila Mista Montana e 2,4 hectares são formados por Floresta Ombrófila Mista Aluvial situada ao longo do riacho que margeia o capão em todo seu limite sul (RONDON NETO et al., 2002).

O bosque situa-se entre as coordenadas $25^{\circ} 26^{\prime} 50^{\prime \prime}$ e $25^{\circ} 27^{\prime} 33^{\prime \prime}$ 'S e $49^{\circ} 14^{\prime}$ ' $6^{\prime \prime}$ e $49^{\circ}$ I $4^{\prime} 33^{\prime \prime} W$, e a altitude do terreno está entre 890 a 915 metros sobre o nível do mar. A área foi submetida a um censo florestal entre o período de julho de 2007 e julho 2008, onde foram medidas e georreferenciadas todas as árvores com $\mathrm{DAP} \geq 10 \mathrm{~cm}$.

\section{Atributos dendrométricos e parâmetros populacionais}

Foram contabilizadas, no censo do fragmento, 9838 árvores de 122 espécies, distribuidas em 47 familias e 81 generos. A densidade total foi de 657 individuos por hectare, com uma área basal de 24,07 $\mathrm{m}^{2} \cdot \mathrm{ha}^{-1}$ (MACHADO et al., 2008). O volume comercial com casca $(V)$ foi estimado em 145,3167 $\mathrm{m}^{3}$ por hectare, por meio de equações volumétricas, definidas para espécies folhosas (I) e para Araucaria angustifolia (Bertol.) Kuntze (2), por Schneider e Finger (2000), onde o DAP é o diâmetro a altura do peito a I,30 m do solo $(\mathrm{cm}) ; \mathrm{h}_{\mathrm{c}}$ é a altura até o ponto de inversão morfológica $(\mathrm{m})$ e $v_{f}$ e $v_{A a}$ são $\circ$ volume das folhosas e de $A$. angustifolia respectivamente $\left(\mathrm{m}^{3}\right)$.

$$
\begin{aligned}
& \mathrm{v}_{\mathrm{f}}=10^{\left[-3,95275+2,04354 \cdot \log (D A P)+0,61461 \cdot \log \left(h_{c}\right)\right]} \\
& \mathrm{v}_{\mathrm{Aa}}=10^{\left[-3,95275+2,04354 \cdot \log (D A P)+0,61461 \cdot \log \left(h_{c}\right)\right]}
\end{aligned}
$$

Para estimativa das alturas de todas as árvores do fragmento, estabeleceu-se uma correlação entre a distribuição diamétrica da população (MACHADO et al., 2009), com a distribuição das alturas de uma amostra de $2500 \mathrm{~m}^{2}$ da área em análise, medidas por meio do hipsômetro Vertex III. Essa correlação foi identificada da seguinte forma:

A probabilidade de um certo valor $\mathrm{x}$ pertencer a um intervalo [a b] qualquer é dada por (3), onde $0 \leq F(x) \leq 1$ :

$$
P(a \leq x \leq b)=\int_{a}^{b} f(x) \cdot d x=F(x)
$$

Seja $f(x)$ uma distribuição densidade de probabilidade qualquer, que descreve as distribuições das variáveis $h_{c}(4)$ e DAP (5). 


$$
\begin{aligned}
& P\left(a \leq h_{c} \leq b\right)=\int_{a}^{b} f\left(h_{c}\right) \cdot d h_{c}=F\left(h_{c}\right) \\
& P(a \leq D A P \leq b)=\int_{a}^{b} f(D A P) \cdot d D A P=F(D A P)
\end{aligned}
$$

Então existe um determinado valor de hc correspondente a um DAP quando $F\left(h_{c}\right)=F(D A P)$.

Dessa forma, foi possivel estimar para qualquer DAP da população, independente dos limites e formas de cada distribuição, utilizando como ferramenta o MS Excel 2007, por meio de seu aplicativo, o Solver.

\section{Simulação dos Métodos de Amostragem}

Em planilha Microsoft Excel 2007, cada árvore medida no fragmento foi considerada como ponto central potencial para se aplicar as metodologias de Bitterlich, Strand e Prodan, ou seja, a origem da unidade amostral. As árvores foram então numeradas sequencialmente. Em seguida, foi elaborado um sorteio sem repetição de 185 árvores, pontos amostrais, das 9838 possíveis, com o uso de uma macro do Microsoft Excel 2007. Na Figura I, é ilustrado o resultado do sorteio dos 185 pontos amostrais.

Cada uma das 185 árvores foi identificada e, a partir dessas, com procedimentos computacionais desenvolvidos para atender aos critérios de amostragem

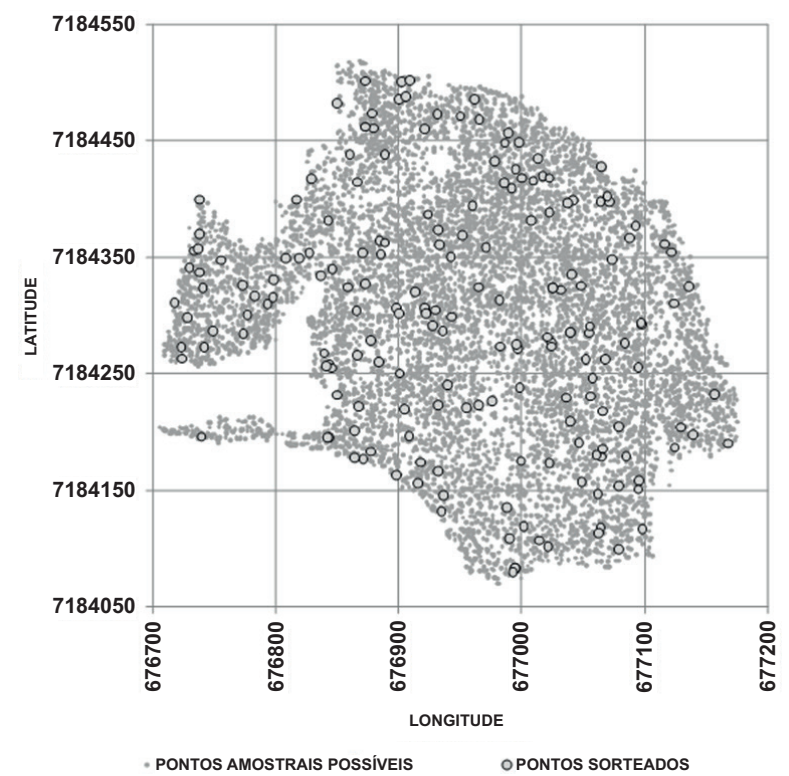

FIGURA 1 Coordenadas geográficas de todas as árvores do fragmento florestal e árvores sorteadas como pontos amostrais.

FIGURE 1 Geographical coordinates of all forest fragment trees and those randomly selected as sample points. de cada método, efetuou-se a seleção das árvores vizinhas. As funções apontaram as árvores cujas distâncias dos seus centros, em relação ao ponto central do ponto de amostragem, eram inferiores ou iguais aos limites requeridos por cada banda, considerando o DAP $(\mathrm{cm})$ de cada árvore.

Nos métodos de Strand e Bitterlich, respectivamente, foram geradas nove simulações de inventário, uma para cada uma das nove bandas do Relascópio de Espelho de Bitterlich, ou seja, I estreita $(F A B=1 / 16), 2$ estreitas $(F A B=1 / 4)$, 3 estreitas (9/16), banda I $(F A B=1)$, banda $I+I$ estreita $(F A B=25 / 16)$, banda $2(F A B=$ $2)$, banda $I+2$ estreitas $(F A B=9 / 4)$, banda $I+3$ estreitas $(F A B=49 / 16)$ e banda $4(F A B=4)$. Para a análise comparativa final, optou-se por usar os resultados gerados pelo FAB que propicia os menores desvios em relação à variável volume, já que essa é a preferida em grande parte dos inventários florestais realizados no país.

Em razão dos procedimentos de campo terem sido efetuados virtualmente, foram consideradas árvores duvidosas (ou marginais) aquelas, cuja distância limite para ser incluída no giro, passava por algum ponto do diâmetro dessa mesma árvore. As seis árvores mais próximas de cada uma das 185 selecionadas como ponto amostral da metodologia de Prodan, foram identificadas por meio de filtros e, para efeito de correção, a sexta árvore foi considerada marginal.

Como eram conhecidas as distâncias dos centros de todas as árvores aos centros de cada ponto amostral, foi possível verificar quais árvores podiam ser incluídas ou não no ponto amostral por meio da equação (6), em que $R_{i}$ é o raio limite de inclusão de uma árvore com diâmetro a altura do peito $d_{i}$ e $F A B$ é o fator de área basal utilizado no método de amotragem. Assim, foi possível realizar também uma simulação de amostragem sem árvores marginais.

$\mathrm{R}_{\mathrm{i}}=\frac{\mathrm{d}_{\mathrm{i}}}{\mathrm{FAB}}$

Para a metodologia de Strand, a linha de amostragem sempre foi originada em cada um dos 185 pontos sorteados, orientada Norte-Sul, percorrendo um comprimento igual a $5 \pi$, onde as coordenadas geográficas das árvores à esquerda de cada linha amostral foram usadas para a determinação dos raios de ação do método. Esses raios foram determinados pelo cálculo da distância do ponto à reta, usando geometria analítica, operacionada em planilha do MS Excel 2007. 
No método de amostragem de Prodan, o efeito da árvore marginal é sempre presente, por isso, não foi possível executar essa metodologia sem considerar as árvores marginais.

\section{Fator de Correção para Árvores Marginais}

Ao se efetuar os procedimentos do método de Bitterlich e Prodan com o relascópio, podem ocorrer árvores duvidosas que, geralmente são consideradas como meia árvore, procedimento esse criticado por Pellico Netto (1994) que sugeriu um fator de correção para essas árvores. Tal fator foi aplicado nos três métodos utilizados neste trabalho e está representado na equação (7), onde $r=$ raio da árvore marginal $(\mathrm{m}) ; R=$ distância do ponto amostral ao centro da árvore marginal $(\mathrm{m}) ; P=$ fator de correção para a árvore marginal.

$P=\frac{\left[\operatorname{sen}^{-1}\left(\frac{\sqrt{R^{2}-r^{2}}}{R}\right)\right] r^{2}+\left[\operatorname{sen}^{-1}\left(\frac{r}{R}\right)\right] R^{2}-\left(\sqrt{R^{2}-r^{2}}\right) r}{\dot{A} r^{2}}$

\section{Comparação dos métodos e dos tratamentos testados}

Os métodos de amostragem utilizados bem como a forma de tratamento da árvore marginal, foram submetidos a testes de comparação de médias e análise de variância (ANOVA), para cada combinação entre método e tratamento e para os diferentes atributos em análise. Foram aplicados o teste de significância de Tukey e ANOVA para blocos casualizados com uma parcela perdida, já que o método de amostragem de Prodan não prevê a ausência da árvore marginal.

\section{RESULTADOS E DISCUSSÃO}

$\mathrm{Na}$ metodologia de Bitterlich, a simulação feita com o fator de área basal correspondente a duas faixas estreitas do relascópio $(F A B=1 / 4)$ foi a que gerou estimativa da área basal e do volume mais próximas das observadas em censo. Já, para o número de árvores por hectate, o melhor $F A B$ foi o correspondente a uma faixa estreita $(1 / 16)$.

Para Strand, o melhor FAB para as estimativas do número de árvores e volume por hectare foi o correspondente a uma faixa estreita $(F A B=1 / 16)$. Já, para a área basal por hectare, o melhor foi o correspondente a três estreitas $(F A B=9 / 16)$.

Segundo o critério de árvores marginais adotado, o número dessas árvores variou de 0 a 4 por ponto amostral, estando presentes em 106 pontos amostrais. Desses, um ponto amostral apresentou 4 árvores duvidosas, 14 apresentaram 3 árvores duvidosas, 25 apresentaram 2 árvores duvidosas e 67 apresentaram apenas I árvore duvidosa.

\section{Estimadores por hectare}

Todos os três métodos utilizados superestimaram o número de árvores por hectare. O método de Strand foi o que mais se aproximou do número de árvores por hectare real (740 árvores·ha $\left.{ }^{-1}\right)$, embora o método de Bitterlich tenha apresentado o menor erro de amostragem relativo, como pode ser observado na Tabela I. Já, Prodan foi o que gerou os valores mais discrepantes.

TABELA 1 Estatísticas do inventário florestal simulado pelos métodos de Bitterlich, Prodan e Strand para estimativa no número de árvores por hectare.

TABLE 1 Statistics from the forest inventory simulated by Bitterlich, Prodan and Strand's methods, to estimate the number of trees per hectare.

\begin{tabular}{cccccccc}
\hline $\begin{array}{c}\text { MÉTODO DE } \\
\text { AMOSTRAGEM }\end{array}$ & \multirow{2}{*}{ Média CV $(\%)$} & $\begin{array}{c}\text { Erro } \\
\text { Padrão }\end{array}$ & $\begin{array}{c}\text { Erro } \\
\text { Abs. }\end{array}$ & $\begin{array}{c}\text { Erro } \\
(\%)\end{array}$ & IA \\
\hline \multirow{2}{*}{ BITTERLICH } & Sem & 766 & 27,09 & 15 & 30 & 3,93 & 28 \\
FAB $=1 / 4$ & Sem P & 773 & 27,11 & 15 & 30 & 3,93 & 28 \\
& Com P & 773 & 27,11 & 15 & 30 & 3,89 & 28 \\
\hline \multirow{2}{*}{ PRODAN } & Sem P & 906 & 43,05 & 29 & 57 & 6,24 & 72 \\
& Com P & 906 & 43,05 & 29 & 57 & 6,24 & 72 \\
\hline \multirow{2}{*}{ STRAND } & Sem & 740 & 36,12 & 20 & 39 & 5,24 & 51 \\
FAB $=1 / 16$ & Sem P & 741 & 36,08 & 20 & 39 & 5,23 & 51 \\
& Com P & 741 & 36,08 & 20 & 39 & 5,23 & 51 \\
\hline
\end{tabular}

$\mathrm{P}$ é o fator de correção da árvore marginal; CV(\%) é o coeficiente de variação; IA é a interação amostral

Esse resultado pode estar ligado à forma de distribuição diâmetrica da floresta e ao número de árvores de pequenas dimensões presentes no fragmento. Machado et al. (2009), estudaram a distribuição diamétrica da área em análise e observaram que a floresta pode ser facilmente modelada por funções exponenciais negativas, indicando que o número de individuos abaixo de $15 \mathrm{~cm}$ de DAP chega a $55 \%$ do total de árvores no fragmentos.

Quando se consideraram árvores marginais como meia árvore e meia árvore corrigida, o número estimado de árvores por hectare foi maior, aumentando a diferença entre o real e o estimado, indicando que, ao realizar os procedimentos em campo, é melhor coletar o diâmetro e a distância do centro da árvore duvidosa até o centro do ponto amostral, para posterior verificação 
da necessidade de sua inclusão do que considerá-la como meia árvore.

Analisando os resultados do inventário com a presença de árvores marginais, observa-se, na Tabela I, que, ao se aplicar o fator de correção às árvores marginais, os resultados praticamente não se alteraram. A média apresentou uma diferença de apenas uma árvore. Já, o erro absoluto não foi alterado, assim como a intensidade amostral necessária em cada método.

É evidente que o número de árvores duvidosas, identificadas neste trabalho, elevou a estimativa média do número de árvores por hectare, porém sua contagem proporcionou um erro de amostragem semelhante quando não se consideram as árvores marginais como meia árvore no inventário.

Portanto, na atividade em campo, é mais prático considerar árvores duvidosas como meia árvore, já que agiliza a execução dos pontos amostrais e, é vantajosa a aplicação do fator $\mathrm{P}$, quando o inventário é efetuado em grandes áreas, porém, para efeitos de precisão, é melhor medir os diâmetros das árvores marginais, assim como a distância de seu centro ao centro do ponto amostral, para, posteriormente, verificar se tal árvore será incluída ou não no referido ponto amostral.

O fator de correção é sensível ao diâmetro da árvore identificada como marginal. Considerando um raio $\mathrm{x}$ qualquer, quanto maior for o diâmetro da árvore, mais relevante será o efeito do fator, portanto, entendese que as árvores marginais consideradas neste trabalho foram, em sua maioria, de diâmetros pequenos.

Esse efeito foi confirmado quando se calculou o diâmetro médio das 9838 árvores medidas no censo e chegou-se ao valor de $18 \mathrm{~cm}$. Assim, o fator $\mathrm{P}$ para uma árvore marginal diâmetro igual ao DAP seria de 0,4996 contra 0,5 se ela fosse considerada como meia. Isso representaria uma diferença de $0,08 \%$ para menos na estimativa da área basal por hectare.

Já, ao se considerar a árvore de maior diâmetro encontrada no censo, como marginal, ter-se-ia uma árvore de $83,5 \mathrm{~cm}$ e, para essa, o fator $P$ seria igual a 0,4984 , o que causaria uma diferença de $0,3 \%$, para menos. Isso indica que o fator de correção teria mais efeito em florestas com diâmetros grandes, o que não é o caso, já que $76 \%$ das árvores do fragmento apresentam diâmetro variando de 10 a $20 \mathrm{~cm}$ e $14 \%$ variando de 20 a $30 \mathrm{~cm}$.

Como a estimativa da área basal por hectare é uma média das áreas basais por hectare estimadas em cada um dos 185 pontos amostrais, o efeito da presença das árvores marginais, corrigidas ou não, foi reduzido, já que em 79 pontos amostrais não foi registrada a presença dessas árvores. Esse fato pode ser observado na Tabela 2, na qual o valor de área basal média, considerando a presença de árvores margnais (sem P). foi ligeiramente maior que o valor gerado quando essas árvores marginais foram corrigidas (com P).

TABELA 2 Estatísticas do inventário florestal simulado pelos métodos de Bitterlich, Prodan e Strand para estimativa da área basal por hectare.

TABLE 2 Statistics of forest inventory simulated by the methods of Bitterlich, Prodan and Strand, to estimate basal area per hectare.

\begin{tabular}{|c|c|c|c|c|c|c|c|}
\hline \multicolumn{2}{|c|}{$\begin{array}{c}\text { MÉTODO DE } \\
\text { AMOSTRAGEM }\end{array}$} & Média & $\begin{array}{l}\text { CV } \\
(\%) \\
\end{array}$ & $\begin{array}{c}\text { Erro } \\
\text { Padrão } \\
\end{array}$ & $\begin{array}{l}\text { Erro } \\
\text { Abs. }\end{array}$ & $\begin{array}{l}\text { Erro } \\
(\%)\end{array}$ & IA \\
\hline \multirow{3}{*}{$\begin{array}{l}\text { BITTERLICH } \\
\text { FAB }=1 / 4\end{array}$} & $\begin{array}{c}\text { Sem } \\
\text { Marginal }\end{array}$ & & 2056 & novio & . & 2,95 & 1 \\
\hline & Sem $P$ & 242 & 20,58 & 0,3621 & 0,7077 & 2,96 & 16,22 \\
\hline & Com P & & 20 & 0,3 & & 2,96 & \\
\hline \multirow{2}{*}{ RODAN } & D & & & & & 0,1 & \\
\hline & Com P & 20 & 56 & 1,21 & 83 & 8,16 & \\
\hline \multirow{3}{*}{$\begin{array}{l}\text { STRAND } \\
=A B=1 / 16\end{array}$} & $\begin{array}{c}\text { Sem } \\
\text { Marginal }\end{array}$ & & 34 & 05 & & 508 & 47 \\
\hline & Sem P & 23, & 34,98 & 0,5934 & 1,1707 & 5,07 & 47,6 \\
\hline & Com P & & & 0 & 07 & 5,07 & 47,6 \\
\hline
\end{tabular}

$\mathrm{P}$ é o fator de correção da árvore marginal; $\mathrm{CV}(\%)$ é o coeficiente de variação; IA é a interação amostral

Ainda, considerando a Tabela 2, vale destacar que $\circ$ método de Bitterlich, sem considerar árvores marginais foi o que gerou a estimativa de área basal por hectare mais próxima da real e o menor erro relativo.

Para as estimativas do volume por hectare, a metodologia de Bitterlich também foi a que gerou os valores mais próximos do real que foi $145,31 \mathrm{~m}^{3} \cdot \mathrm{ha}^{-1}$. Já Strand foi o método que gerou as estimativas mais distantes do valor real, e o método de Prodan apresentou os maiores erros de amostragem, como pode ser observado na Tabela 3. Como essas estimativas são dependentes do número de árvores por hectare, o desempenho apresentado por Prodan para N.ha- ${ }^{-1}$ foi similar aos apresentados para $\mathrm{V}_{\left(\mathrm{m}^{3}\right)} \cdot \mathrm{ha}^{-1}$.

Já, quando se comparam as árvores marginais, contadas como meia árvore (sem P) e as contadas como meia árvore corrigida (com P) nota-se que as estimativas sem correção (sem $P$ ) apresentaram uma pequena diferença para mais. Tal fato já foi constatado por Pellico Netto (1994), o que o fez propor o procedimento de correção para as árvores marginais, visando à solução do problema da superestimação. 
TABELA 3 Estatísticas do inventário florestal simulado pelos métodos de Bitterlich, Prodan e Strand para estimativa do volume por hectare.

TABLE 3 Statistics of forest inventory simulated by the methods of Bitterlich, Prodan and Strand, to estimate volume per hectare.

\begin{tabular}{|c|c|c|c|c|c|c|c|}
\hline \multicolumn{2}{|c|}{$\begin{array}{c}\text { MÉTODO DE } \\
\text { AMOSTRAGEM }\end{array}$} & Média & $\begin{array}{l}\mathrm{CV} \\
(\%)\end{array}$ & $\begin{array}{c}\text { Erro } \\
\text { Padrão }\end{array}$ & $\begin{array}{l}\text { Erro } \\
\text { Abs. }\end{array}$ & $\begin{array}{l}\text { Erro } \\
(\%)\end{array}$ & IA \\
\hline \multirow{3}{*}{$\begin{array}{c}\text { BITTERLICH } \\
\text { FAB }=1 / 4\end{array}$} & $\begin{array}{c}\text { Sem } \\
\text { Marginal }\end{array}$ & 1 & 21,61 & 2,2740 & 4,4440 & 3,1 & 17 \\
\hline & Sem $P$ & 143,0856 & 21,61 & 2,2736 & 4,4433 & 3,10 & 17,89 \\
\hline & Com P & 143 & 21,61 & 2,2736 & 4,4433 & 3,10 & 17,89 \\
\hline \multirow{2}{*}{ PRODAN } & Sem P & 150 & 67,52 & 7,4879 & 14,7732 & 9,7 & 77,48 \\
\hline & Com P & 150,7016 & 67,50 & 7,4790 & 14,7556 & 9,79 & 177,36 \\
\hline \multirow{3}{*}{$\begin{array}{l}\text { STRAND } \\
F A B=1 / 16\end{array}$} & $\begin{array}{c}\text { Sem } \\
\text { Marginal }\end{array}$ & 28 & 37,44 & 3,7629 & 7,4239 & 5,43 & 54,55 \\
\hline & Sem P & 136,8448 & 37,42 & 3,7652 & 7,4285 & 5,43 & 54,51 \\
\hline & Com P & 136,8447 & 37,42 & 3,7652 & 7,4285 & 5,43 & 54,51 \\
\hline
\end{tabular}

P é o fator de correção da árvore marginal; $\mathrm{CV}$ (\%) é o coeficiente de variação; IA é a interação amostral

\section{Comparação das médias}

A comparação das médias estimadas pelos tratamentos (sem árvore marginal, com $\mathrm{P}$ e sem $\mathrm{P}$ ), bem como a comparação dos métodos para estimativa do $\mathrm{N} \cdot h \mathrm{ha}^{-1}, \mathrm{G} \cdot \mathrm{ha}^{-1} \mathrm{e} \mathrm{V} \cdot \mathrm{ha}^{-1}$ indicaram que não há diferença significativa entre os tratamentos, entretanto os métodos de amostragem utilizados diferem estatisticamente entre si, como pode ser visto na Tabela 4, 5 e 6 .

Esses resultados evidenciam que a contagem da árvore marginal como meia árvore ou a aplicação do $P$ na mesma, não resulta em uma diferença significativa entre as médias obtidas por cada tratamento dentro dos métodos de amostragem utilizados. Entretanto, os métodos de amostragem de Bitterlich, Strand e Prodan diferem ao nível de 5 e $1 \%$ de probabilidade, resultado inerente a forma de inclusão da árvore marginal no cálculo do estimador por unidade de área independente da variável em análise.

$\mathrm{Na}$ Tabela 7, estão presentes os atributos estimados por cada combinação de método de amostragem e tratamento da árvore marginal, bem como os resultados da comparação de médias feitas pelo teste de Tukey ao nível de $1 \%$ de probabilidade.

$\mathrm{Na}$ Tabela 7, fica evidente que há uma diferença significativa entre as médias obtidas por cada método, independente da variável em análise. Entretanto, o delta cálculado para cada atributo não identifica que há uma diferença entre os tratamentos praticados, independente do método de amostragem utilizado.
TABELA 4 ANOVA da estimativa do número de árvores por hectare pelos diferentes métodos de amostragem e tratamentos estudados.

TABLE 4 ANOVA of the estimated number of trees per hectare by different sampling methods and treatments studied.

\begin{tabular}{llllll}
\hline FONTE DA \\
VARIAÇÃO
\end{tabular}$\quad \mathrm{SQ} \quad \mathrm{GL} \quad \mathrm{MQ} \quad \mathrm{F} \quad$ valor- $P \frac{\mathrm{F} \text { crítico }}{5 \%}$

Tratamentos $30,9461 \quad 2 \quad 15,4731 \quad 1,9328 \quad 0,2888 \quad 9,55 \quad 30,82$

$\begin{array}{llllll}\text { Métodos de } & & & \end{array}$

Resíduos $\quad 24,0171 \quad 3 \quad 8,00569$

Total $46028,0 \quad 7$

TABELA 5 ANOVAda estimativa de área basal por hectare pelos diferentes métodos de amostragem e tratamentos estudados.

TABLE 5 ANOVA of the estimated basal area per hectare by different sampling methods and treatments studied.

\begin{tabular}{lccccccc}
\hline $\begin{array}{l}\text { FONTE DA } \\
\text { VARIAÇÃO }\end{array}$ & SQ & GL & MQ & F & Valor- $P$ & \multicolumn{2}{c}{$F$ crítico } \\
\hline Tratamentos & 0,0296 & 2 & 0,0148 & 1,9226 & 0,2901 & 9,55 & 30,82 \\
Métodos de & 20,099 & 2 & 10,049 & 1306,6 & 0,0000 & 9,55 & 30,82 \\
Amostragem & & & & & & & \\
Resíduos & 0,0231 & 3 & 0,0077 & & & \\
\hline Total & 20,152 & 7 & & & & \\
\hline
\end{tabular}

TABELA 6 ANOVA da estimativa de volume por hectare pelos diferentes métodos de amostragem e tratamentos estudados.

TABLE 6 ANOVA of the estimated volume per hectare by different sampling methods and treatments studied.

\begin{tabular}{llllll}
\hline FONTE DA & $S Q$ & $G L$ & $M Q$ & $F$ & valor-P $\frac{F \text { crítico }}{5 \%} \quad 1 \%$
\end{tabular}

Tratamentos $0,007320,0036 \quad 0,663218 \quad 0,5774 \quad 9,55 \quad 30,82$

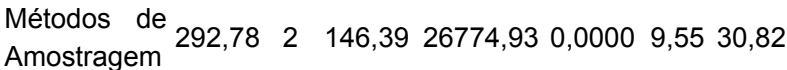
Resíduos $\quad 0,0164 \quad 3 \quad 0,0055$

Total $\quad 292,80 \quad 7$

Esse resultado reforça que os métodos de amostragem de área variável possuem uma diferença significativa na obtenção de estimadores por unidade de área. É possivel afirmar que o efeito da ocorrência de árvores marginais bem como a sua forma de inclusão na parcela de área variável geram diferenças pouco expressivas na estimativa dos atributos por unidade de área. 
TABELA 7 Estimadores de número de árvores, área basal e volume por hectare nos diferentes métodos de amostragem utilizados, bem como o agrupamento

TABLE 7 das médias pelo teste de Tukey.

Estimators of number of trees, basal area and volume per hectare in the different sampling methods used, as well as clustering of means by Tukey test.

\begin{tabular}{ccccc}
\hline \multirow{2}{*}{$\begin{array}{c}\text { MÉTODO DE } \\
\text { AMOSTRAGEM }\end{array}$} & \multirow{2}{*}{ TRATAMENTO } & \multicolumn{3}{c}{ ESTIMADORES } \\
\cline { 3 - 5 } & & N/ha & G/ha & V/ha \\
\hline \multirow{2}{*}{ BITTERLICH } & Sem Marginal & $766^{\mathrm{a}}$ & $24,0376^{\mathrm{a}}$ & $143,1222^{\mathrm{a}}$ \\
& Sem F $_{\text {CAM }}$ & $773^{\mathrm{a}}$ & $24,2583^{\mathrm{a}}$ & $143,0856^{\mathrm{a}}$ \\
& Com F $_{\text {CAM }}$ & $773^{\mathrm{a}}$ & $24,2582^{\mathrm{a}}$ & $143,0842^{\mathrm{a}}$ \\
\hline \multirow{2}{*}{ STRAND } & Sem Marginal & $740^{\mathrm{b}}$ & $23,0501^{\mathrm{b}}$ & $136,7128^{\mathrm{b}}$ \\
& Sem $\mathrm{F}_{\text {CAM }}$ & $741^{\mathrm{b}}$ & $23,0724^{\mathrm{b}}$ & $136,8448^{\mathrm{b}}$ \\
& Com $\mathrm{F}_{\text {CAM }}$ & $741^{\mathrm{b}}$ & $23,0724^{\mathrm{b}}$ & $136,8447^{\mathrm{b}}$ \\
\hline \multirow{2}{*}{ PRODAN } & Sem $\mathrm{F}_{\text {CAM }}$ & $906^{\mathrm{c}}$ & $26,6938^{\mathrm{c}}$ & $150,8293^{\mathrm{c}}$ \\
& Com $\mathrm{F}_{\text {CAM }}$ & $906^{\mathrm{c}}$ & $26,6747^{\mathrm{c}}$ & $150,7016^{\mathrm{c}}$ \\
\hline Diferença Mínima Significativa $\Delta^{* *}=17$ & 0,5315 & 0,4483 \\
\hline
\end{tabular}

\section{CONCLUSÕES}

método de Bitterlich apresentou os menores erros de amostragem na estimativa dos parametros populacionais área basal e volume por hectare, já, o método de Strand resultou em melhor desempenho na estimativa do número de árvores por hectare;

Há uma diferença significativa entre os métodos de amostragem de Bitterlich, Strand e Prodan, quanto à estimativa do número de árvores, área basal e volume por hectare. Destaque ao método de amostragem de Prodan que superestima independente da variável ou do tratamento da árvore marginal;

A predominânica de diâmetros pequenos na base de dados usada, não proporcionou uma efetiva correção quando aplicou-se o fator $\mathrm{P}$;

As árvores marginais contadas parcialmente após a aplicação do fator $\mathrm{P}$, não geraram uma melhora expressiva nos estimadores populacionais, a ponto de se diferenciar, satisfatoriamente, da estimativa gerada com as árvores marginais contadas como meia árvore ou sem a sua influência.

\section{REFERÊNCIAS}

BITTERLICH, W. Die Winkelzahlprobe. Allgemeine Forst und Holzwirtschaftliche Zeitung, Wein, v. 59, n. I/2, p. 4-5, 1948.

MACHADO, S. do A.; AUGUSTYNCZIK, A. D. L.; NASCIMENTO, R. G. M.; TÉO, S. J.; MIGUEL, E. P.; FIGURA, M. A.; SILVA, L. C. R. da. Funções de distribuiç̧ão diamétrica em um fragmento de Floresta Ombrófila Mista. Ciência Florestal, Santa Maria, v. 39, n. 8, p. 2428-2434, 2009.

MACHADO, S. do A.; NASCIMENTO, R. G. M.; AUGUSTYNCZIK, A. D. L.; TÉO, S. J. Parâmetros fitossociológicos de um fragmento de Floresta Ombrófila Mista. In: SIMPÓSIO LATINO-AMERICANO SOBRE MANEJO FLORESTAL, 4., 2008, Santa Maria. Anais... Santa Maria: UFSM/CCR/PPGEF, 2008. p. 433-438.

PÉLLICO NETTO, S. Fator de correção para árvores marginais em métodos de amostragem com seleção probabilística proporcional a um tamanho. Cerne, Lavras, v. I, n. I, p. 73-77, 1994.

PRODAN, M. Punktstichprobe fur die forsteinrichtung. Der Forst- und Holzwirt, Hannover, v. 23, n. I I, p. 225-226, 1968.

RONDON NETO, R. M.; KOZERA, C.; ANDRADE, R. do R. de; CECY, A. T.; HUMMES, P. A.; FRITZSONS, E.; CALDEIRA, M. V. W.; MACIEL, M. de N. M.; SOUZA, M. K. F. de. Caracterização florística e estrutural de um fragmento de Floresta Ombrófila Mista, em Curitiba, PR, Brasil. Floresta, Curitiba, v. 32, n. I, p. 3-16, jan./jun. 2002.

SCHNEIDER, P. R.; FINGER, C. A. G. Manejo sustentado de florestas inequiâneas heterogêneas. Santa Maria: UFSC, 2000. 195 p.

STRAND, L. Sampling for volume along a line. Meddelelser fra Det norske Skogforsoksvesen, Oslo, v. 5I, p. 327$331,1958$. 